\title{
Stem Cells for the Treatment of Neurological Disorders
}

Loss of neurons and glial cells cause disorders like Parkinson's disease, stroke, spinal injury and multiple sclerosis.

In recent years neurons and glial cell have been generated successfully from stem cells in culture fuelling efforts to develop stem cell based transplantation therapies for human patients.

In Bangladesh a centre for stem cell research and therapy has been established at Dhaka Medical College Hospital under neurosurgery department. We hope that this centre will be contributing a lot for many Bangladeshi people to avail this opportunity.

Stem cell based therapy has raised particular interest in the treatment of Parkinson's disease, stroke. Head injury, spinal cord injury, Huntington's disease, Amyotrophic lateral sclerosis and Multiple sclerosis.

Steady progress supports the hope that stem cell based therapies to restore and preserve function in the brain and spinal cord can be developed. 